\title{
4,5-Dichloro-1,2,3-dithiazolium Chloride (Appel's Salt): Reactions with N-nucleophiles.
}

\author{
Ana M. Cuadro and Julio Alvarez-Builla ${ }^{b_{*}}$ \\ aDepartment of Chemistry, Imperial College of Science, Technology and Medicine, London SW7 2AY, England. \\ bDepartamento de Química Orgánica, Universidad de Alcalá. 28871 Alcalá de Henares. Madrid. Spain.
}

\begin{abstract}
Different N-nucleophiles have been reacted with 4,5-dichloro-1,2,3-dithiazolium chloride (Appel's Salt), producing imines containing the 1,2,3-dithiazole ring.
\end{abstract}

Appel's Salt 1 is the most studied derivative of the 1,2,3-dithiazolium system, due to its ready preparation from chloroacetonitrile and disulfur dichloride. ${ }^{1}$ Early chemistry was devoted to the reactions with arylamines, phenol and active methylene compounds, always giving nucleophilic substitutions on the chlorine of the 5-position, and yielding compounds $2-5 .{ }^{1}$ The chemistry has been recently reviewed, in relation to the field of heterocycles with polysulfur-nitrogen bonds, ${ }^{2}$ describing useful conversions of compounds 5 into benzothiophene derivatives, and 4 into benzothiazole and benzoxazole derivatives or alternatively into cyanoimidoyl chlorides. ${ }^{3}$ More recently, a method using 1 to produce esters under mild conditions has been described. ${ }^{4}$

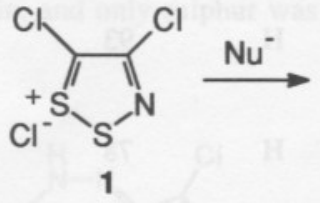<smiles></smiles>

Fig. 1

$$
\begin{aligned}
& \text { 2, } X=S \\
& \text { 3, } X=O \\
& \text { 4, } X=N A r \\
& \text { 5, } X=R_{1} R_{2} C
\end{aligned}
$$

In the present paper we have studied the reactions of Appel's salt with hydrazine derivatives and amino heterocycles (shown in Fig. 1), in order to open routes to new heterocyclic systems.

In the course of the investigation into the reaction of 1 with hydrazines using the reported conditions ${ }^{2}$, it was found that the use of base (triethylamine, Hunig's base, pyridine or lutidine), even when the addition of hydrazines $(6 \mathrm{a}, \mathrm{b}$, and $\mathrm{c})$ was carried out at low temperature, produced a complex reaction mixture, from which the only product characterized was the thione $\mathbf{2}$. Nothing is known about the 
mechanism, but the extra sulfur must come from another molecule of starting material. ${ }^{5}$ The formation of the thione 2 seems to take place, in the presence of the hydrazine and a base such as pyridine, when the reaction of the nucleophile with $\mathbf{1}$ is slow. The attack of the base at the sulfur atom then becomes a competing reaction resulting in the formation of 2 . Conversely, with more reactive nucleophiles such as aniline the thione $\mathbf{2}$ is not observed, unless the aniline contains electron withdrawing substituents.

After the initial observations, we concluded that the optimum conditions to avoid thione formation were when the reaction of $\mathbf{1}$ was carried out without base, at room temperature under nitrogen, in DCM or THF, and with slow addition of hydrazines 6 (a-c ). Thus, derivatives 7 were obtained in good yield as salts. However, experiments with benzoylhydrazine, p-toluenesulfonylhydrazide and $\mathrm{N}$-aminopthalimide led to neutral derivatives $\mathbf{8}(\mathbf{a}-\mathbf{c})($ Table1).<smiles>C[As]1CC(Cl)=NS1</smiles><smiles>[R6]N([R2])[NH+]([R6])C</smiles><smiles>[R]N([R])N([R5])c1[s+]snc1Cl</smiles><smiles>[R]N([R])/N=c1\ssnc1Cl</smiles>

Fig. 2

Table 1. Reactions of 1 with hydrazines.

\begin{tabular}{ccccc} 
Comp. No & $\mathrm{R}_{1}$ & $\mathrm{R}_{2}$ & $\mathrm{R}_{3}$ & Yield (\%) \\
\hline 7a & $\mathrm{C}_{6} \mathrm{H}_{5}$ & $\mathrm{C}_{6} \mathrm{H}_{5}$ & $\mathrm{H}$ & 40 \\
7b & $\mathrm{C}_{6} \mathrm{H}_{5}$ & $\mathrm{H}$ & $\mathrm{C}_{6} \mathrm{H}_{5}$ & $49^{\mathrm{a}}$ \\
7c & $\mathrm{C}_{6} \mathrm{H}_{5}$ & $\mathrm{H}$ & $\mathrm{COCH}_{3}$ & 70 \\
8a & $\mathrm{C}_{6} \mathrm{H}_{5} \mathrm{CO}$ & $\mathrm{H}$ & $\mathrm{H}$ & 93 \\
8c & $\mathrm{p}-\mathrm{CH}_{3} \mathrm{C}_{6} \mathrm{H}_{4} \mathrm{SO}_{2}$ & $\mathrm{H}$ & $\mathrm{H}$ & 78 \\
& & & &
\end{tabular}

aproduct isolated as hydrochloride. 
Derivatives 7 (a-c) were light sensitive, and gradually decomposed by loss of hydrogen chloride. As they seemed promising intermediates for a cycloaddition process we tested $7 \mathbf{b}$ under suitable conditions, in the presence of dipolarophiles. Attempts were made hoping that any initially formed $\mathrm{N}$-ylide would react with dimethyl acetylenedicarboxylate (DMAD) or N-methylmaleimide (NMM). However, decomposition was observed, with the imine 4 being the only identifiable product isolated (Fig. 3).

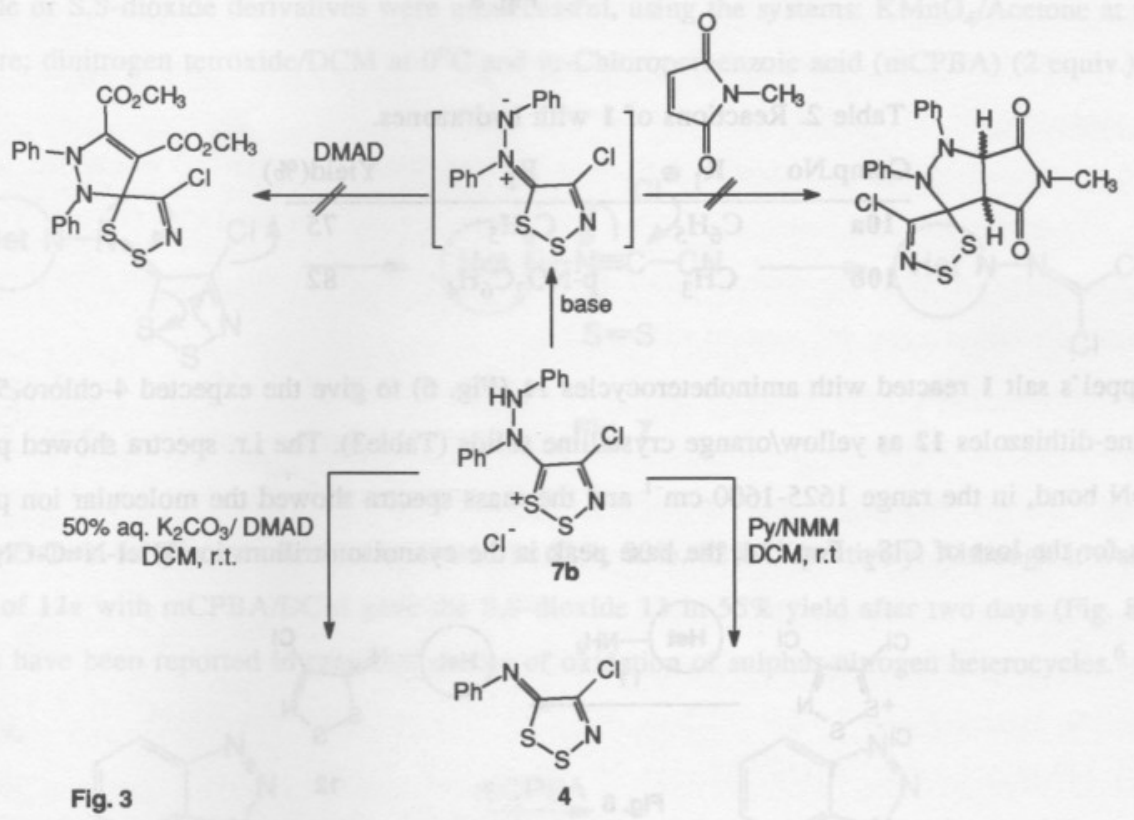

We tried further reactions with the stable derivative 8a, hoping that it would undergo transformations giving 2-cyano-5-phenyl-[1,3,4]oxadiazole (Fig. 4), via the intermediate spirocompound, by loss of $\mathrm{S}_{2}$ and hydrochloric acid. ${ }^{2}$ The expected compound was not obtained when it was refluxed in xylene for $30 \mathrm{~min}$, and only sulphur was isolated.

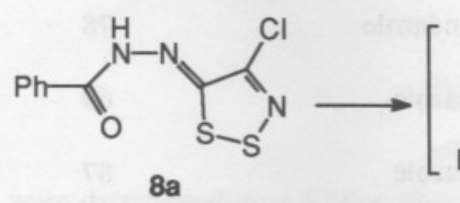

$8 \mathrm{a}$

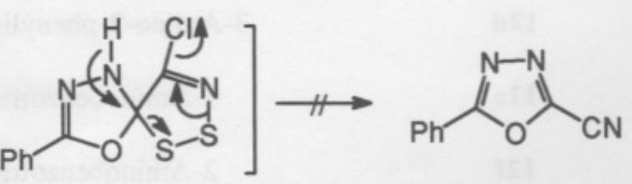

Fig. 4

The reaction with other nucleophiles such as hydrazones $\mathbf{9}$ gave the corresponding derivatives $\mathbf{1 0}$ in good yield (Table 2) without the need for added base. 


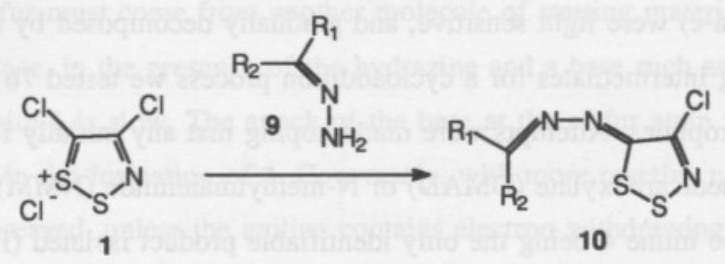

Fig. 5

Table 2. Reactions of $\mathbf{1}$ with hydrazones.

\begin{tabular}{cccc} 
Comp.No & $\mathrm{R}_{1}$ & $\mathrm{R}_{2}$ & Yield(\%) \\
\hline $10 \mathrm{a}$ & $\mathrm{C}_{6} \mathrm{H}_{5}$ & $\mathrm{C}_{6} \mathrm{H}_{5}$ & 75 \\
$10 \mathrm{~b}$ & $\mathrm{CH}_{3}$ & p- $-\mathrm{NO}_{2} \mathrm{C}_{6} \mathrm{H}_{4}$ & 82
\end{tabular}

Appel's salt 1 reacted with aminoheterocycles 11 (Fig. 6) to give the expected 4-chloro-5heteroimine-dithiazoles 12 as yellow/orange crystalline solids (Table3). The i.r. spectra showed peaks, due to the $\mathrm{C}=\mathrm{N}$ bond, in the range $1625-1600 \mathrm{~cm}^{-1}$ and the mass spectra showed the molecular ion plus the fragments for the loss of $\mathrm{ClS}_{2}$. For 12d, the base peak is the cyanoisonitrilium ion $[\mathrm{Het}-\mathrm{N}=\mathrm{C}-\mathrm{CN}]^{+}$.<smiles>ClC1[Si][SH]2CCCC2C1Cl</smiles>

1

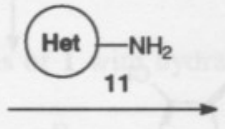

Fig. 6<smiles>ClC1NNC(=Nc2ccccc2)S1</smiles>

12

Table 3. Reactions of 1 with Aminoheterocycles.

Comp.

12a

12b

$12 \mathrm{c}$

12d

$12 \mathrm{e}$

$12 f$

$12 \mathrm{~g}$

$12 \mathrm{~h}$
Aminoheterocycle

3-Aminopyrazole

5-Amino-3,4-diphenyl-1-p-tolylpyrazole

2-Amino-[1,3,4] thiadiazole

3-Amino-2-phenylindazole

1-Aminobenzotriazole

2-Aminobenzotriazole

2-Aminobenzimidazole

2-Aminobenzothiazole
Yield (\%)

67

69

87

72

49 
Compounds $8 \mathrm{c}$ and $12 \mathrm{e}$ were thermally stable, resisting prolonged reflux in solvents such as xylene or DMF. They were also relatively stable when heated at $200^{\circ} \mathrm{C}$ or $250^{\circ} \mathrm{C}$ for 5 minutes without solvent. When $8 \mathrm{c}$ and $12 \mathrm{e}$ were pyrolysed at $550^{\circ} \mathrm{C} / 1.2 \mathrm{mbar}$, starting material $(80$ and $70 \%)$ was recovered, with no sign of the expected chloroimine ${ }^{2}$ (Fig.7).

Oxidation of heterocyclic imines $8 \mathrm{c}$ and 12e were investigated, and attempts to convert $8 \mathbf{c}$ into the S-oxide or S,S-dioxide derivatives were unsuccessful, using the systems: $\mathrm{KMnO}_{4} /$ Acetone at room temperature; dinitrogen tetroxide/DCM at $0^{\circ} \mathrm{C}$ and $\mathrm{m}$-Chloroperbenzoic acid (mCPBA) (2 equiv.)/DCM at

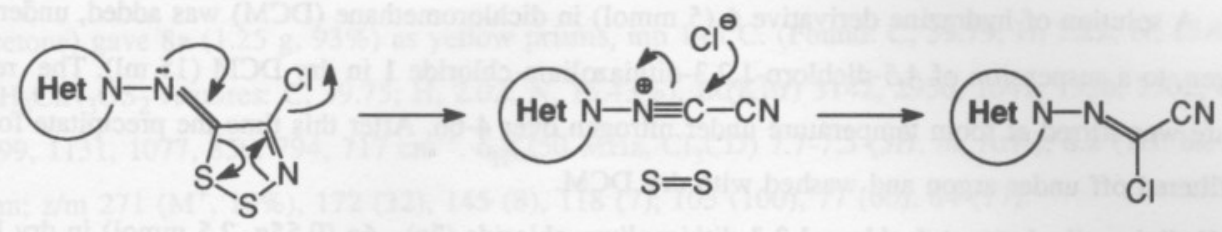

Fig. 7

room temperature. In all cases $8 \mathrm{c}$ was recovered in $62 \%, 80 \%, 82 \%$ respectively. Although it was slow, treatment of $12 \mathrm{e}$ with mCPBA/DCM gave the S,S-dioxide 13 in 55\% yield after two days (Fig. 8). Similar oxidations have been reported in previous studies of oxidation of sulphur-nitrogen heterocycles. ${ }^{6}$<smiles>Clc1nss/c1=N\n1nnc2ccccc21</smiles>

$12 e$

$$
\underset{\mathrm{DCM}, \mathrm{m.t.}}{\stackrel{\mathrm{mCPBA}}{\longrightarrow}}
$$

Fig. 8<smiles>O=S1(=O)N=C(Cl)/C(=N\n2nnc3ccccc32)S1</smiles>

In summary, the reaction of Appel's salt with either hydrazine derivatives or aminoheterocycles produced imines as described for arylamines. Development of processes for conversion of 7, 8, 10, and 12 into heterocyclic systems is underway.

\section{EXPERIMENTAL}

Mps were determined on a Köfler apparatus and are uncorrected. IR spectra were recorded either on Perkin-Elmer 1710 FT or 1310 instruments. ${ }^{1} \mathrm{H}$ NMR spectra were recorded on a JEOL GSX 270 (270 $\mathrm{MHz})$, a Bruker WM250 (250MHz), or a Varian Unity $300(300 \mathrm{MHz})$ spectrometers. ${ }^{13} \mathrm{CNMR}$ spectra were recorded on a Bruker WM250 $(62.9 \mathrm{MHz})$ and a Varian Unity $300(75.429 \mathrm{MHz})$. Low resolution 
mass spectra was recorded on a VG Micromass 7070B instrument or a Hewlett-Packard 5988A, in the electron impact mode at $70 \mathrm{eV}$, using a direct insertion probe. High resolution $\mathrm{EI}$ and FAB mass spectra were recorded on a VG Analytical ZAB-E instrument. Column chromatography was on silica gel (60 Merck, $230-400 \mathrm{mesh})$. Light petroleum refers to the fraction b.p. $40-60^{\circ} \mathrm{C}$. Satisfactory microanalyses were obtained for all new compounds described, within $0.4 \%$ error. The starting heterocyclic precursors were obtained using previously described methods. $1,2,7-9$

Reaction of 4,5-dichloro-1,2,3-dithiazolium chloride with hydrazines.

General procedure for the preparation of derivatives 7 and 8 .

A solution of hydrazine derivative $6(5 \mathrm{mmol})$ in dichloromethane (DCM) was added, under nitrogen, to a suspension of 4,5-dichloro-1,2,3-dithiazolium chloride 1 in dry DCM (15 ml). The reaction mixture was stirred at room temperature under nitrogen over 4-6h. After this time the precipitate formed was filtered off under argon and washed with dry DCM.

5 -( $N, N$-diphenylhydrazine)-4-chloro-1,2,3-dithiazolium chloride $(7 \mathrm{a}) .6 \mathrm{a}(0.55 \mathrm{~g}, 2.5 \mathrm{mmol})$ in dry DCM (2 $\mathrm{ml}$ ) was added dropwise to a suspension of 4,5-dichloro-1,2,3-dithiazolium chloride $1(0.521 \mathrm{~g}, 2.5 \mathrm{mmol})$ in dry DCM $(10 \mathrm{ml})$ under nitrogen. The mixture was stirred at room temperature for $6 \mathrm{~h}$. Then, the resulting precipitate was filtered under argon and washed with dry DCM $(2 \times 15 \mathrm{ml})$ to give $7 \mathrm{a}(0.356 \mathrm{~g}$, $40 \%$ ) as a dark blue solid, $\mathrm{mp} 110^{\circ} \mathrm{C}$. (Found: $\mathrm{C}, 47.34 ; \mathrm{H}, 3.49 ; \mathrm{N}, 12.01 . \mathrm{C}_{14} \mathrm{H}_{11} \mathrm{Cl}_{2} \mathrm{~N}_{3} \mathrm{~S}_{2}$ requires: $\mathrm{C}$, 47.19; H, 3.11; N, 11.79\%). IR(KBr) 2864, 2588, 1600, 1558, 1516, 1493, 1194, 1113, 1090, 1031,744 $\mathrm{cm}^{-1} . \delta_{\mathrm{H}}(250 \mathrm{MHz}, \mathrm{DMSO}) 7.6-6.9(10 \mathrm{H}, \mathrm{m}, \mathrm{ArH}) ; 4.6(1 \mathrm{H}, \mathrm{bs},) \mathrm{ppm} . \mathrm{z} / \mathrm{m} \mathrm{319}\left(\mathrm{M}^{+}-\mathrm{HCl}, 32 \%\right), 220(10)$, 169 (57), 149 (42), 84 (29), 77 (18), 38 (33), 36 (100).

5 -( $N, N^{\prime}$-Diphenylhydrazine)-4-chloro-1,2,3-dithiazolium chloride (7b). Following the above procedure $\mathrm{N}, \mathrm{N}$ '-diphenylhydrazine $6 \mathrm{~b}(0.92 \mathrm{~g}, 5 \mathrm{mmol})$ was added to a suspension of $1(1.042 \mathrm{~g}, 5 \mathrm{mmol})$ in dry DCM $(15 \mathrm{ml})$ under nitrogen atmosphere and the mixture stirred for $6 \mathrm{~h}$, after which the precipitate was filtered off, washed with DCM $(2 \times 20 \mathrm{ml})$ and dried to afford $7 \mathrm{~b}(0.967 \mathrm{~g}, 49 \%)$ as green prisms, mp 112$114^{\circ} \mathrm{C}$. (Found: $\mathrm{C}, 43.13 ; \mathrm{H}, 2.72 ; \mathrm{N}, 11.17 . \mathrm{C}_{14} \mathrm{H}_{11} \mathrm{Cl}_{2} \mathrm{~N}_{3} \mathrm{~S}_{2} \cdot \mathrm{HCl}$ requires: $\mathrm{C}, 42.81 ; \mathrm{H}, 3.07 ; \mathrm{N}, 10.69 \%$ ). IR(KBr) $3418,2855,1586,1544,1485,1454,1340,1201,775 \mathrm{~cm}^{-1} . \delta_{\mathrm{H}}(250 \mathrm{MHz}, \mathrm{DMSO}) 7.9-7.2(10 \mathrm{H}$, $\mathrm{m}, \mathrm{ArH}) ; 6,2(1 \mathrm{H}, \mathrm{bs}) \mathrm{ppm} . \delta_{\mathrm{C}}(62.9 \mathrm{MHz}$; DMSO) $154.4,147.9,136.8,130.3,129.7,127.7,127.5,125.9$, 123.6, 123.2, 120.1, 119.1 ppm. $z / m$ 319(M+2 $-2 \mathrm{HCl}, 1 \%), 272$ (2), 228 (16), 184 (24), 167 (14), 126 (16), 103 (10), 93 (14), 77 (53), 64 (100), 38 (23), 36 (72). m/z (FAB, MNBA matrix) $356\left(\mathrm{M}^{+}-\mathrm{Cl}, 0.5 \%\right), 320$ (6), 229 (55), 185 (100), 77 (56).

5-[N-A cetyl-N'-phenylhydrazine)-4-chloro-1,2,3-dithiazolium chloride (7c). Following the above procedure, 1-phenyl-2-acetylhydrazine $6 \mathrm{c}(0.375 \mathrm{~g}, 2.5 \mathrm{mmol})$ in dry DCM $(2 \mathrm{ml})$ was added to $1(0.521 \mathrm{~g}, 2.5$ mmol) in dry DCM $(10 \mathrm{ml})$ and the mixture was stirred at room temperature for $1 \mathrm{~h}$ under nitrogen, to give $7 \mathrm{c}(0.563 \mathrm{~g}, 70 \%)$ as a pale green solid, $\mathrm{mp} 111^{\circ} \mathrm{C}$. (Found: $\mathrm{C}, 37.97 ; \mathrm{H}, 3.06 ; \mathrm{N}, 13.48$. $\mathrm{C}_{10} \mathrm{H}_{9} \mathrm{Cl}_{2} \mathrm{~N}_{3} \mathrm{OS}_{2}$ requires: C, 37.27; $\mathrm{H}, 2.81 ; \mathrm{N}, 13.48 \%$ ). IR(KBr) $3477,3413,1699,1639,1619,1495$, 
$1455,1279,1245,1184,819,715 \mathrm{~cm}^{-1} . \delta_{\mathrm{H}}(250 \mathrm{MHz}, \mathrm{DMSO}) 7.65(1 \mathrm{H}, \mathrm{bs}) ; 7.15-6.7(5 \mathrm{H}, \mathrm{m}, \mathrm{ArH})$; 2.15 (3H, s, Me) ppm. z/m 285 ( $\left.\mathrm{M}^{+}-\mathrm{HCl}, 3 \%\right), 193$ (12), 186 (13), 176 (8), 160 (8), 150 (13), 132 (20), 108 (37), 91(85), 77 (40), 64 (100), 36 (40). m/z (FAB, MNBA matrix) 286 (M+-Cl, 28\%), 193 (100), 168 (35), 151(90), 109 (12), 77 (26).

Benzoic acid (4-chloro-[1,2,3]dithiazol-5-ylidene)-hydrazide (8a). A solution of benzoylhydrazine $(0.680 \mathrm{~g}$, $5 \mathrm{mmol}$ ) in dry DCM was added dropwise to a stirred suspension of 4,5-dichloro-1,2,3-dithiazolium chloride $\mathbf{1}(1.042 \mathrm{~g}, 5 \mathrm{mmol})$ in DCM $(15 \mathrm{ml})$. After stirring at room temperature for $8 \mathrm{~h}$, the resulting precipitate was filtered off and washed with DCM $(3 \times 15 \mathrm{ml})$, then dried in vacuo. Recrystallisation (acetone) gave $8 \mathrm{a}(1.25 \mathrm{~g}, 93 \%)$ as yellow prisms, $\mathrm{mp} 145^{\circ} \mathrm{C}$. (Found: $\mathrm{C}, 39.79 ; \mathrm{H}, 2.22 ; \mathrm{N}, 15.46$. $\mathrm{C}_{9} \mathrm{H}_{6} \mathrm{ClN}_{3} \mathrm{OS}_{2}$ requires: $\mathrm{C}, 39.75 ; \mathrm{H}, 2.02 ; \mathrm{N}, 15.43 \%$ ). IR(KBr) 3142, 2956, 1641, 1556, 1302, 1290 , $1199,1131,1077,859,794,717 \mathrm{~cm}^{-1} . \delta_{\mathrm{H}}\left(250 \mathrm{MHz}, \mathrm{Cl}_{3} \mathrm{CD}\right) 7.7-7.5(5 \mathrm{H}, \mathrm{m}, \mathrm{ArH}) ; 8.2(1 \mathrm{H}, \mathrm{bs}, \mathrm{NH})$ ppm; z/m 271 (M+1 19\%), 172 (32), 145 (8), 118 (7), 105 (100), 77 (60), 64 (17).

Toluene-4-sulfonic acid (4-Chloro-[1,2,3]dithiazol-5-ylidene)-hydrazide (8b). 4-toluenesulfonylhydrazide $(0.46 \mathrm{~g}, 2.5 \mathrm{mmol})$ was added to a suspension of 4,5-dichloro-[1,2,3]dithiazolium chloride 1 ( $0.52 \mathrm{~g}, 2.5$ $\mathrm{mmol})$ in dry THF $(8 \mathrm{ml})$. The reaction mixture was stirred at room temperature for $4 \mathrm{~h}$. The solvent was removed in vacuo and the residue washed with DCM $(20 \mathrm{ml})$ followed by chromatography of the residue. Eluting with DCM gave $8 \mathrm{~b}(0.62 \mathrm{~g}, 78 \%)$ as a yellow solid, $\mathrm{mp} 159-160^{\circ} \mathrm{C}$. (Found: $\mathrm{C}, 33.63 ; \mathrm{H}, 2.30 ; \mathrm{N}$, 12.85. $\mathrm{C}_{9} \mathrm{H}_{8} \mathrm{ClN}_{3} \mathrm{O}_{2} \mathrm{~S}_{3}$ requires: $\left.\mathrm{C}, 33.59 ; \mathrm{H}, 2.50 ; \mathrm{N}, 13.05 \%\right)$. IR(KBr) 3414, 3134, 1593, 1556, 1371 , $1332,1202,1165,1087,985,891,819,805,741 \mathrm{~cm}^{-1} . \delta_{\mathrm{H}}\left(270 \mathrm{MHz}, \mathrm{Cl}_{3} \mathrm{CD}\right) 7.90(2 \mathrm{H}, \mathrm{d}, \mathrm{J} 7 \mathrm{~Hz}, \mathrm{ArH})$; 7.35 (2H, d, J 7Hz, ArH); 2.45 (3H, s, ArMe) ppm. z/m 321( M $\left.^{+}, 0.5 \%\right) 246$ (76), 139 (13), 123 (90), 91 (100).

2-(4-chloro-[1,2,3]dithiazol-5-ylideneamino)-isoindole-1,3-dione (8c). $\mathrm{N}$-Aminopthalimide $(0.81 \mathrm{~g}, 5 \mathrm{mmol})$ was added in portions to a stirred suspension of 4,5-dichloro-1,2,3-dithiazolium chloride 1 (1.042 g, 5 $\mathrm{mmol})$ in dry DCM $(15 \mathrm{ml})$. After stirring at room temperature for $4 \mathrm{~h}$ under nitrogen, the resulting precipitate was filtered and washed with dry DCM $(3 \times 5 \mathrm{ml})$. Recrystallization from DMF-MeOH gave 8c $\left(1.19 \mathrm{~g}, 80 \%\right.$ ) as yellow prisms, mp $275-276^{\circ} \mathrm{C}$. (Found: $\mathrm{C}, 40.31 ; \mathrm{H}, 1.30 ; \mathrm{N}, 14.13 . \mathrm{C}_{10} \mathrm{H}_{4} \mathrm{ClN}_{3} \mathrm{~S}_{2} \mathrm{O}_{2}$ requires: C, 40.31; H, 1.35; N, 14.11\%); $\mathrm{IR}(\mathrm{KBr}) 1788,1704,1366,1351,1399,1199,1106,1081,892$, $705 \mathrm{~cm}^{-1} . \delta_{\mathrm{H}}\left(250 \mathrm{MHz}\right.$; DMSO) $7.90-7.84(4 \mathrm{H}, \mathrm{m}, \mathrm{ArH}) ; \delta_{\mathrm{C}}(62.9 \mathrm{MHz}, \mathrm{DMSO}) 166.0,162.1,142.7$, 134.9, 130.4, 123.7 ppm. z/m 297 (M+, 58\%), 262 (13), 198 (100), 104 (35), 90 (13), 76 (39).

Reaction of 4,5-dichloro1,2,3-dithiazolium chloride with hydrazones.

$N$-B enzhydrylidene- $N^{\prime}$-(4-chloro-[1,2,3]dithiazol-5-ylidene)-hydrazine (10a). 4,5-dichloro-1,2,3-dithiazolium chloride $1(1.042 \mathrm{~g}, 5 \mathrm{mmol})$ was added to a solution of benzophenonehydrazone $9 \mathrm{a}(0.981 \mathrm{~g}, 5 \mathrm{mmol})$ in dry THF $(300 \mathrm{ml})$ under nitrogen. The solution was stirred at room temperature for $3 \mathrm{~h}$, then THF was removed under reduced pressure to afford a red oil, which was purified by chromatography (DCM as eluent) to give $10 \mathrm{a}(1.230 \mathrm{~g}, 75 \%)$ as a red oil (Found: $\mathrm{C}, 54.38 ; \mathrm{H}, 2.93 ; \mathrm{N}, 12.47 . \mathrm{C}_{15} \mathrm{H}_{10} \mathrm{ClN}_{3} \mathrm{~S}_{2}$ 
requires: C, 54.29; H, 3.03; N, 12.66\%). IR(film) 3430, 3058, 1586, 1558, 1444, 1323, 1205, 891, 785, $695 \mathrm{~cm}^{-1} . \delta_{\mathrm{H}}\left(270 \mathrm{MHz}, \mathrm{Cl}_{3} \mathrm{CD}\right) 7.80-7.35(10 \mathrm{H}, \mathrm{m}, \mathrm{ArH}) \mathrm{ppm} . \mathrm{z} / \mathrm{m} 331\left(\mathrm{M}^{+}, 0.5 \%\right) 182(39), 153(38)$, 125 (19), 105 (74), 93 (22), 77 (40), 64 (100).

$N$-(4-chloro-[1,2,3]dithiazol-5-ylidene- $N^{\prime}-[1-(4-n i t r o p h e n y l)$-ethylidene]hydrazine (10b). A solution of 4nitroacetophenonehydrazone $9 \mathrm{~b}(0.89 \mathrm{~g}, 5 \mathrm{mmol})$ in THF $(5 \mathrm{ml})$, was added to a suspension of $1(1.042 \mathrm{~g}$, $5 \mathrm{mmol})$ in dry THF $(10 \mathrm{ml})$. After stirring at room temperature for $1 \mathrm{~h}$, the THF was removed in vacuo. The residue was triturated with ether $(3 \times 5 \mathrm{ml})$ and the resulting precipitate filtered. Recrystallisation in acetone gave $10 \mathrm{~b}(0.32 \mathrm{~g}, 82 \%)$ as orange crystals, $\mathrm{mp} 147^{\circ} \mathrm{C}$. (Found: $\mathrm{C}, 38.25 ; \mathrm{H}, 2.17 ; \mathrm{N}, 18.09$. $\mathrm{C}_{10} \mathrm{H}_{7} \mathrm{ClN}_{4} \mathrm{O}_{2} \mathrm{~S}_{2}$ requires: C, 38.15; $\mathrm{H}, 2.24 ; \mathrm{N}, 17.88 \%$ ). IR(KBr) $3414,1578,1510,1487,1338,1302$, $1202,891,855,787,730 \mathrm{~cm}^{-1} . \delta_{\mathrm{H}}\left(270 \mathrm{MHz}, \mathrm{Cl}_{3} \mathrm{CD}\right) 8.30$ (2H, d, J $\left.8 \mathrm{~Hz}, \mathrm{ArH}\right) ; 8.13(2 \mathrm{H}, \mathrm{d}, \mathrm{ArH}) ; 2.6(\mathrm{~s}$, 3H, Me) ppm. z/m 314 (M+, 76\%), 284 (13), 215 (15), 163 (36), 149 (23), 117 (100), 76 (47).

Reaction of 4,5-dichloro-1,2,3-dithiazolium chloride with heterocyclic amines.

(4-chloro-[1,2,3]dithiazol-5-ylidene)-(1H-pyrazol-3-yl)-amine (12a). A solution of 3-aminopyrazole 11a $(0.207 \mathrm{~g}, 2.5 \mathrm{mmol})$ in DCM $(1 \mathrm{ml})$ was added to a stirred suspension of 4,5-dichloro-1,2,3-dithiazolium chloride $(0.521 \mathrm{~g}, 2.5 \mathrm{mmol})$ in dry DCM $(7 \mathrm{ml})$. After stirring at room temperature for $5 \mathrm{~h}$ the DCM was removed and the residue purified by column chromatography (DCM, as eluent) to give $12 \mathrm{a}(0.365 \mathrm{~g}, 67 \%)$ as a yellow solid, $\mathrm{mp} 179.5-180^{\circ} \mathrm{C}$. (Found: $\mathrm{C}, 27.22 ; \mathrm{H}, 1.51 ; \mathrm{N}, 25.37 . \mathrm{C}_{5} \mathrm{H}_{3} \mathrm{ClN}_{4} \mathrm{~S}_{2}$ requires: $\mathrm{C}, 27.46$; $\mathrm{H}, 1.38 ; \mathrm{N}, 25.62 \%)$. IR(KBr) $3225,1541,1351,1161,877,763 \mathrm{~cm}^{-1} . \delta_{\mathrm{H}}(300 \mathrm{MHz}, \mathrm{DMSO}) 13.21(1 \mathrm{H}$, bs $\mathrm{NH}) ; 7.90(1 \mathrm{H}, \mathrm{s}) ; 6.48(1 \mathrm{H}, \mathrm{s}) \mathrm{ppm} . \delta_{\mathrm{C}}(75 \mathrm{MHz}, \mathrm{DMSO}) 154.4,153.5,147.6,131.2,101.9 \mathrm{ppm} . \mathrm{z} / \mathrm{m}$ $218\left(\mathrm{M}^{+}, 30 \%\right), 83$ (44), 157 (29), 119 (16), 93 (21), 70 (28), 64 (100).

(4-chloro-[1,2,3]dithiazol-5-yliden)-(4,5-diphenyl-2-p-tolyl-2H-pyrazol-3-yl)-amine (12b). 5-amino-3,4diphenyl-1-p-tolyl-pyrazole ${ }^{7} 11 \mathrm{~b}(0.405 \mathrm{~g}, 1.25 \mathrm{mmol})$ and $1(0.260 \mathrm{~g}, 1.5 \mathrm{mmol})$ were stirred at room temperature in dry DCM $(4 \mathrm{ml})$ for $2 \mathrm{~h}$. Removal of the DCM in vacuo, followed by chromatography of the resulting residue (DCM as eluent) gave $12 \mathrm{~b}(0.489 \mathrm{~g}, 85 \%)$ as a yellow-orange crystalline solid, $\mathrm{mp}$ $158^{\circ} \mathrm{C}$. (Found: $\mathrm{C}, 62.85 ; \mathrm{H}, 3.56 ; \mathrm{N}, 11.98 . \mathrm{C}_{24} \mathrm{H}_{17} \mathrm{ClN}_{4} \mathrm{~S}_{2}$ requires: $\mathrm{C}, 62.53 ; \mathrm{H}, 3.71 ; \mathrm{N}, 12.15 \%$ ).

IR(KBr) $3420,3061,2920,1577,1512,1368,1167,871,778,734,700 \mathrm{~cm}^{-1} . \delta_{\mathrm{H}}\left(250 \mathrm{MHz}, \mathrm{Cl}_{3} \mathrm{CD}\right) 7.75$ $(1 \mathrm{H}, \mathrm{d}, \mathrm{J} 8 \mathrm{~Hz} \mathrm{ArH}) ; 7.55-7.48(2 \mathrm{H}, \mathrm{m}, \mathrm{ArH}) ; 7.35-7.20(10 \mathrm{H}, \mathrm{m}, \mathrm{ArH}) ; 2.40(3 \mathrm{H}, \mathrm{s}, \mathrm{Me}) \mathrm{ppm} . \delta_{\mathrm{C}}(62.9$ $\left.\mathrm{MHz}, \mathrm{Cl}_{3} \mathrm{CD}\right) 159.5,150.0,148.0,144.7,136.9,136.8,133.0,132.4,129,3,129.2,128.9,128.5,128.2$, $127.9,127.3,123.4,110.2,21.06$ ppm. $z / m 460$ (M+ $100 \%), 427$ (15), 367 (92), 361 (74), 334 (19), 325 (45), 149 (11), 91(29), 89 (38), 77 (18).

(4-chloro-[1,2,3]dithiazol-5-ylidene)-([1,3,4]thiadiazol-2-yl)-amine (12c). 2-Amino-[1,3,4-]hiadiazole 11c $(0.252 \mathrm{~g}, 2.5 \mathrm{mmol})$ was added to a suspension of $1(0.521 \mathrm{~g}, 2.5 \mathrm{mmol})$ in dry DCM $(7 \mathrm{ml})$. After stirring at room temperature for $4 \mathrm{~h}$ the residue was isolated and purified by column chromatography (DCM, 100\%) to give $12 \mathrm{c}(0.38 \mathrm{~g}, 70 \%)$ as a yellow solid, $\mathrm{mp} 234-235^{\circ} \mathrm{C}$. (Found: $\mathrm{C}, 20.41 ; \mathrm{H}, 0.70 ; \mathrm{N}$, $23.80 ; \mathrm{C}_{4} \mathrm{HClN}_{4} \mathrm{~S}_{3}$ requires: $\mathrm{C}, 20.22 ; \mathrm{H}, 0.42 ; \mathrm{N}, 23.59 \%$ ). IR(KBr) $3416,1509,1469,1402,1168,902$ 
$\mathrm{cm}^{-1} \cdot \delta_{\mathrm{H}}(300 \mathrm{MHz}, \mathrm{DMSO}) 9.59(\mathrm{~s}, 1 \mathrm{H}) . \delta_{\mathrm{C}}(75 \mathrm{MHz}, \mathrm{DMSO}) 163.7,154.5,147.5,130.3 \mathrm{ppm} . \mathrm{z} / \mathrm{m} 236$ $\left(\mathrm{M}^{+}, 27\right), 201(100), 102$ (36), 70 (40), 64 (58).

(4-chloro-[1,2,3]dithiazol-5-ylidene)-(2-phenyl-2H-indazol-3-yl)-amine (12d). A solution of 3-amino-2phenylindazole ${ }^{8} 11 \mathrm{~d}(0.172 \mathrm{~g}, 0.82 \mathrm{mmol})$ was added to a suspension of 4,5-dichloro-1,2,3-dithiazolium chloride $(0.170 \mathrm{~g}, 0.82 \mathrm{mmol})$ in dry DCM $(3 \mathrm{ml})$. After stirring at room temperature for $2 \mathrm{~h}$, the solution was concentrated under reduced pressure, and the residual oil was treated with DCM-light petroleum (1:1) giving a yellow solid which was purified by column chromatography to give $12 \mathrm{~d}(0.220 \mathrm{~g}, 78 \%)$, $\mathrm{mp} 136$ $138^{\circ} \mathrm{C}$. (Found: $\mathrm{C}, 52.62, \mathrm{H}, 2.48 ; \mathrm{N}, 15.74 . \mathrm{C}_{15} \mathrm{H}_{9} \mathrm{ClN}_{4} \mathrm{~S}_{2}$ requires: $\mathrm{C}, 52.24 ; \mathrm{H}, 2.63 ; \mathrm{N}, 16.24 \%$ ). IR(KBr) 3022, 2917, 1626, 1606, 1593, 1472, 1334, 767, $694 \mathrm{~cm}^{-1} . \delta_{\mathrm{H}}(250 \mathrm{MHz}, \mathrm{DMSO}) 9.04$ (1H, d, J $7 \mathrm{~Hz} \mathrm{ArH}) ; 8.24$ (1H, t, ArH); $8.14(1 \mathrm{H}, \mathrm{d}, \operatorname{ArH}) ; 8.04(1 \mathrm{H}, \mathrm{t}, \mathrm{ArH}) ; 7.92-7.76(5 \mathrm{H}, \mathrm{m}, \operatorname{ArH}) \mathrm{ppm} . \mathrm{z} / \mathrm{m} 245$ $\left(\mathrm{M}^{+}-\mathrm{ClS}_{2}, 100 \%\right), 219(2), 102$ (7), 91(2), 77 (11).

Benzotriazol-1-yl-(4-chloro-[1,2,3]dithiazol-5-ylidene)-amine (12e). A solution of 1-aminobenzotriazole ${ }^{9}$ 11e $(0.67 \mathrm{~g}, 5 \mathrm{mmol})$ in THF (15 ml), was added to a suspension of $1(1.042 \mathrm{~g}, 5 \mathrm{mmol})$ in dry DCM (15 $\mathrm{ml}$ ) and the mixture was stirred at room temperature for $6 \mathrm{~h}$. Filtration gave a solid which was purified by column chromatography (DCM as eluent) and then recrystallized from DMF-MeOH to give $12 \mathrm{e}(0.92 \mathrm{~g}$, $69 \%$ ) as yellow prisms mp $244-245^{\circ} \mathrm{C}$. (Found: $\mathrm{C}, 35.52 ; \mathrm{H}, 1.30 ; \mathrm{N}, 25.74 . \mathrm{C}_{8} \mathrm{H}_{4} \mathrm{ClN}_{5} \mathrm{~S}_{2}$ requires: $\mathrm{C}$, 35.62 ; H, 1.49; N, 25.96\%); IR(KBr) 3257, 2520, 1624, 1612, 1531, 1495, 1448, 1242, 1191, 1154, 907, $890,769,743 \mathrm{~cm}^{-1} . \delta_{\mathrm{H}}(250 \mathrm{MHz}, \mathrm{DMSO}) 8.20(1 \mathrm{H}, \mathrm{d}, \mathrm{J} 8 \mathrm{~Hz}) ; 7.95(1 \mathrm{H}, \mathrm{d}) ; 7.75(1 \mathrm{H}, \mathrm{t}) ; 7.63(1 \mathrm{H}, \mathrm{t})$ ppm. $\delta_{\mathrm{C}}(62.9 \mathrm{MHz}, \mathrm{DMSO}) 154.4,144.5,144.4,129.3,128.9,126.1,119.8,110.8 \mathrm{ppm} . \mathrm{m} / \mathrm{z} 269\left(\mathrm{M}^{+}\right.$, 27\%), 178 (79), 134 (60), 105 (69), 77 (100).

Benzotriazol-2-yl-(4-chloro-[1,2,3]dithiazol-5-ylidene)-amine (12f). Following the above procedure, 2aminobenzotriazole ${ }^{9} 11 \mathrm{f}(0.134 \mathrm{~g}, 1 \mathrm{mmol})$ was stirred with $1(0.208 \mathrm{~g}, 1 \mathrm{mmol})$ in DCM $(6 \mathrm{ml})$ at room temperature for $2 \mathrm{~h}$. Work-up of the mixture gave $12 \mathrm{f}(0.23 \mathrm{~g}, 87 \%)$ as a yellow crystalline solid $\mathrm{mp}$ $287^{\circ} \mathrm{C}$. (Found: $\mathrm{C}, 35.76 ; \mathrm{H}, 1.38 ; \mathrm{N}, 25.73 . \mathrm{C}_{8} \mathrm{H}_{4} \mathrm{ClN}_{5} \mathrm{~S}_{2}$ requires: $\mathrm{C}, 35.62 ; \mathrm{H}, 1.49 ; \mathrm{N}, 25.96 \%$ ). $\operatorname{IR}(\mathrm{KBr}) 1516,1489,1485,1437,1402,1269,1237,1223,1184,897,801,737 \mathrm{~cm}^{-1} . \delta_{\mathrm{H}}(250 \mathrm{MHz}$, DMSO) 8.10-7.90 (2H, m); 7.65-7.55 (2H, m) ppm. z/m 269 (M+, 100\%), 234 (87), 160 (22), 102 (26), 90 (23).

Benzimidazol-2-yl-(4-chloro-[1,2,3]dithiazol-5-ylidene)-amine (12g). Following the above procedure, 2aminobenzimidazole $11 \mathrm{~g}(0.332 \mathrm{~g}, 2.5 \mathrm{mmol})$ was stirred with $1(0.521 \mathrm{~g}, 2.5 \mathrm{mmol})$ in dry DCM $(7 \mathrm{ml})$ at room temperature for $4 \mathrm{~h}$. Removal of the DCM followed by chromatography of the resulting residue $(100 \% \mathrm{DCM})$ gave $12 \mathrm{~g}(0.487 \mathrm{~g}, 72 \%)$ as an orange-yellow crystalline solid, mp $230^{\circ} \mathrm{C}$. (Found: $\mathrm{C}, 39.98$; $\mathrm{H}, 1.98 ; \mathrm{N}, 20.59 . \mathrm{C}_{9} \mathrm{H}_{5} \mathrm{ClN}_{4} \mathrm{~S}_{2}$ requires: $\mathrm{C}, 40.22 ; \mathrm{H}, 1.87$; N, 20.84\%). IR(KBr) 3404, 1529, 1483, 1438 , $1416,1167,884,800,742 \mathrm{~cm}^{-1} . \delta_{\mathrm{H}}(300 \mathrm{MHz}, \mathrm{DMSO}) 12.9(\mathrm{~s}, 1 \mathrm{H}-\mathrm{NH}) ; 7.67(1 \mathrm{H}, \mathrm{d} \mathrm{J} 7 \mathrm{~Hz}) ; 7.45(1 \mathrm{H}$, d); 7.29-7.22 (2H, m) ppm. $z / m$ 268(M+ $\left.\mathrm{M}^{+}, 40 \%\right), 233$ (M+Cl, 100), 201 (7), 175 (17), 169 (10), 143 (31), $116(10), 90(13)$. 
Benzothiazol-2-yl-(4-chloro-[1,2,3]dithiazol-5-ylidene)-amine (12h) . 2-aminobenzothiazole $11 \mathrm{~h}(0.375 \mathrm{~g}$, $2.5 \mathrm{mmol}$ ) was added in portions to a stirred suspension of 4,5 ,-dichloro-[1,2,3]dithiazolium chloride 1 $(0.521 \mathrm{~g}, 2.5 \mathrm{mmol})$ in dry DCM $(7 \mathrm{ml})$. After stirring at room temperature for $4 \mathrm{~h}, \mathrm{DCM}$ was removed in vacuo. The residue was purified by column chromatography (DCM/petroleum ether) to give $12 \mathrm{~h}(0.35 \mathrm{~g}$, $49 \%$ ) as an orange solid, $\mathrm{mp} 153-154^{\circ} \mathrm{C}$. (Found: $\mathrm{C}, 37.50 ; \mathrm{H}, 1.66 ; \mathrm{N}, 14.82 . \mathrm{C}_{9} \mathrm{H}_{4} \mathrm{ClN}_{3} \mathrm{~S}_{3}$ requires: $\mathrm{C}$, $37.82 ; \mathrm{H}, 1.41 ; \mathrm{N}, 14.70 \%)$. IR(KBr) $3413,1507,1480,1446,1418,1311,1250,1159,924,755 \mathrm{~cm}^{-1}$. $\delta_{\mathrm{H}}(300 \mathrm{MHz}, \mathrm{DMSO}) 8.69(1 \mathrm{H}, \mathrm{d}, \mathrm{J} 7.8 \mathrm{~Hz}) ; 7.92(1 \mathrm{H}, \mathrm{d}) ; 7.53(1 \mathrm{H}, \mathrm{t}, \mathrm{J} 7.5 \mathrm{~Hz}) ; 7.42(1 \mathrm{H}, \mathrm{t}) \mathrm{ppm}$. $\delta_{\mathrm{C}}(75 \mathrm{MHz}, \mathrm{DMSO}) 168.9,163.2,148.8,147.7,134.6,126.9,125.3,122.6,121.6 \mathrm{ppm} . \mathrm{z} / \mathrm{m} 285\left(\mathrm{M}^{+}\right.$, $38 \%), 250$ ( $\left.\mathrm{M}^{+}-\mathrm{Cl}, 100\right), 192$ (12), 186 (5), 160 (62), 134 (18), 108 (38).

Reaction of 12e with m-Chloroperbenzoic acid (mCPBA)

Benzotriazol 1-yl-(4-chloro-2,2-dioxo-[1,2,3]dithiazol-5-ylidene-amine (13). $12 \mathrm{e}(151 \mathrm{mg}, 0.56 \mathrm{mmol})$, mCPBA (168 mg, $0.82 \mathrm{mmol})$ and DCM $(60 \mathrm{ml})$ were stirred at $0^{\circ} \mathrm{C}$ for $1 \mathrm{~h}$, followed by stirring for a further $24 \mathrm{~h}$ at room temperature. A final portion of mCPBA $(168 \mathrm{mg}, 0.82 \mathrm{mmol})$ was added and the reaction mixture stirred for another $24 \mathrm{~h}$, until all the starting material had been consumed as observed by tlc. Purification by column chromatography (DCM as eluent) gave $13(92 \mathrm{mg}, 55 \%)$ as an orange-yellow solid, mp 171-2 ${ }^{\circ} \mathrm{C}$. (Found: $\mathrm{C}, 31.88 ; \mathrm{H}, 1.23 ; \mathrm{N}, 23.12 . \mathrm{C}_{8} \mathrm{H}_{4} \mathrm{ClN}_{5} \mathrm{O}_{2} \mathrm{~S}_{2}$ requires: $\mathrm{C}, 31.84 ; \mathrm{H}, 1.43 ; \mathrm{N}$, $23.21 \%)$. IR(KBr) $1574,1446,1149,1047,879,751 \mathrm{~cm}^{-1} . \delta_{\mathrm{H}}\left(270 \mathrm{MHz}, \mathrm{Cl}_{3} \mathrm{CD}\right) 8.12(1 \mathrm{H}, \mathrm{d} \mathrm{J} 8 \mathrm{~Hz}) ; 7.80$ $(1 \mathrm{H}, \mathrm{d}) ; 7.65(1 \mathrm{H}, \mathrm{t}) ; 7.52(1 \mathrm{H}, \mathrm{t}) \mathrm{ppm} . \mathrm{z} / \mathrm{m} \mathrm{301}\left(\mathrm{M}^{+}, 3 \%\right), 285\left(\mathrm{M}^{+}-16,23\right), 269\left(\mathrm{M}^{+}-32,3\right), 174(17), 155$ (32), 146 (100), 136 (28), 108 (22), 93 (39), 76 (73).

\section{ACKNOWLEDGEMENTS}

The authors wish to express sincere gratitude to Prof. C.W. Rees for his support and advice during the stay of A.M.C at the I.C. and to the Ministerio de Educación y Ciencia for providing a grant.

\section{REFERENCES}

1. Appel, R.; Janssen, H.; Siray, M.; Knoch, F. Chem. Ber. 1985, 118, 1632.

2. Rees, C.W. J. Heterocyclic Chem. 1992, 29, 583.

3. English, R. F.; Rees, C. W. Imperial College, unpublished work.

4. Folmer, J. J.; Weinreb, S. M. Tetrahedron Lett. 1993, 34, 2737.

5. Gray, M. A.; Rees, C. W. J. Chem. Soc. Perkin Trans.1. 1993, 3077.

6 Dunn, P.J.; Rees, C.W. J.Chem.Soc.Perkin Trans.1. 1989, 2485.

7. Smith, P.A.S.; Breen, G.J.W.; Hajek, M.K.; Awana, D.V.C. J.Org.Chem. 1970, 35, 2215.

8. Partridge, M; Stevens, M.F.G. J.Chem.Soc. 1964, 3663.

9. Campbell, C.D.; Rees, C. W. J. Chem. Soc. (C). 1969, 742. 